\title{
ON PARAMETRIC VECTOR OPTIMIZATION VIA METRIC REGULARITY OF CONSTRAINT SYSTEMS
}

\author{
M. DUREA \\ Faculty of Mathematics, "Al. I. Cuza" University, \\ Bd. Carol I, nr. 11, 700506 - Iaşi, Romania, \\ e-mail: durea@uaic.ro \\ R. STRUGARIU \\ Department of Mathematics, "Gh. Asachi" Technical University, \\ Bd. Carol I, nr. 11, 700506 - Iaşi, Romania, \\ e-mail: rstrugariu@tuiasi.ro
}

\begin{abstract}
Some metric and graphical regularity properties of generalized constraint systems are investigated. Then, these properties are applied in order to penalize (in the sense of Clarke) various scalar and vector optimization problems. This method allows us to present several necessary optimality conditions in solid constrained vector optimization.
\end{abstract}

Keywords: set-valued mappings $\cdot$ metric regularity $\cdot$ scalar and vector optimization

Mathematics Subject Classification (2010): 90C30 $\cdot 49 \mathrm{~J} 53 \cdot 54 \mathrm{C} 60$

\section{Introduction and motivation}

In theory of optimization, a large literature is dedicated to the branch of parametric optimization problems with equilibrium constraints and this is one of the reasons which motivates the many efforts made in the last decades in studying the variational systems (appearing as constraint systems in different mathematical programs) in their own right and involving growing generality. We quote here only the important works of Robinson [15], Dontchev and Rockafellar [4], Mordukhovich [12] for comprehensive discussions and historical facts.

In this paper we firstly aim to underline some aspects of these topics by presenting in a simple manner some optimization-related motivational facts leading to two types of metric regularity for fairly general variational systems. Then we survey, complete and extend some results previously obtained by the authors. Lastly, we get optimality conditions for some vector mathematical programs, by the use of the regularity of constraints system and the power of Mordukhovich's generalized differentiation theory.

Let $X$ be a Banach space. In this setting, $B(x, r)$ and $D(x, r)$ denote the open and the closed ball with center $x$ and radius $r$, respectively. Sometimes we write $\mathbb{D}_{X}, \mathbb{B}_{X}$ and $\mathbb{S}_{X}$ for the closed, the open unit ball and the unit sphere of $X$, respectively. If $x \in X$ and $A \subset X$, one defines the distance 
from $x$ to $A$ as $d(x, A):=\inf \{\|x-a\| \mid a \in A\}$. As usual, we use the convention $d(x, \emptyset)=\infty$. The distance function to $A$ is defined as $d_{A}=d(\cdot, A)$. For a non-empty set $A \subset X$, we put int $A$ for the topological interior. When we work on a product space, we consider the sum norm, unless otherwise stated.

Consider now a multifunction $F: X \rightrightarrows Y$ between the Banach spaces $X$ and $Y$. The domain and the graph of $F$ are denoted respectively by

$$
\operatorname{Dom} F:=\{x \in X \mid F(x) \neq \emptyset\}
$$

and

$$
\operatorname{Gr} F=\{(x, y) \in X \times Y \mid y \in F(x)\} .
$$

If $A \subset X$ then $F(A):=\bigcup_{x \in A} F(x)$. The inverse set-valued map of $F$ is $F^{-1}: Y \rightrightarrows X$ given by $F^{-1}(y)=\{x \in X \mid y \in F(x)\}$. Recall that $F$ is said to be inner semicontinuous at $(\bar{x}, \bar{y}) \in \operatorname{Gr} F$ if for every open set $D \subset Y$ with $\bar{y} \in D$, there exists a neighborhood $U \in \mathcal{V}(\bar{x})$ such that for every $x \in U, F(x) \cap D \neq \emptyset$ (where $\mathcal{V}(\bar{x})$ stands for the system of the neighborhoods of $\bar{x}$ ). On the other hand, $F$ is said to be Lipschitz-like around $(\bar{x}, \bar{y})$ with constant $L>0$ if there exist two neighborhoods $U \in \mathcal{V}(\bar{x}), V \in \mathcal{V}(\bar{y})$ such that, for every $x, u \in U$,

$$
F(x) \cap V \subset F(u)+L\|x-u\| \mathbb{D}_{Y} .
$$

Let us begin our study with the presentation of the scalar case. Our motivation is provided by some parametric optimization problems (in our notations the set of parameters is $P$ and it is initially taken as a topological space). In the simplest case of a scalar objective defined by a parametric function $f: X \times P \rightarrow \mathbb{R}$, we look at the problem

$$
\left(P_{S}\right): \min f(x, p) \text { subject to } 0 \in H(x, p),
$$

where $H: X \times P \rightrightarrows Y$ is a multifunction which defines a generalized constraints system by the relation $0 \in H(x, p)$. One can find in literature this kind of problems under the generic term of "optimization with equilibrium constraints". The implicit set-valued map $S: P \rightrightarrows X$ associated to $H$ is

$$
S(p)=\{x \in X \mid 0 \in H(x, p)\} .
$$

In fact, if for any fixed $p \in P$ one considers the problem of minimizing the function $f(\cdot, p)$ with the constraint $0 \in H(x, p)$, the set $S(p)$ is the feasible set of this problem. In general, the treatment of the problem would involve calculus associated to the set-valued map $S$, but this becomes a rather delicate situation since, in general, $S$ and the associated coderivatives could be hard to compute. One possibility would be as in [6, Theorem 5.3], and this involves implicit multifunction theorems, in the line of those presented in Section 3 of this paper.

Let us consider first the case of parametric paradigm, i.e. the case where one considers the optimality with respect to $x$ and for some fixed values of parameters. In this respect, let us take $M \subset P$ as a nonempty set. We say that $\bar{x} \in X$ is a solution for $\left(P_{S}\right)$ with respect to $M$ if $\bar{x} \in \bigcap_{p \in M} S(p)$ and for every $p \in M$ there exists $\varepsilon_{p}>0$ s.t. for every $x \in B\left(\bar{x}, \varepsilon_{p}\right) \cap S(p)$, one has

$$
f(\bar{x}, p) \leq f(x, p)
$$


This definition actually says that for every fixed $\widetilde{p} \in M, \bar{x}$ is a local solution for the scalar nonparametric problem

$$
\min f(x, \widetilde{p}) \text { subject to } 0 \in H(x, \widetilde{p}) \text {. }
$$

In order to illustrate this definition, let us consider $f: \mathbb{R} \times \mathbb{R} \rightarrow \mathbb{R}, f(x, p)=x^{2}+p^{2}$ and $H: \mathbb{R} \times \mathbb{R} \rightrightarrows \mathbb{R}, H(x, p)=-x-p+1+\mathbb{R}_{+}$. Now, $\bar{x}=0$ is a solution for this $\left(P_{S}\right)$ with respect to $M:=[-1,+\infty)$.

Now, we use the Clarke penalization technique for Lipschitz functions (see [3, Proposition 2.4.3]) in our context. For this, we need to define a concept of equi-lipschitzianity for the parametric objective function. In the above notation, for a positive $L$, one says that the function $f$ is $L$-Lipschitz at $\bar{x} \in X$ with respect to $M \subset P$ if for every $p \in M$ there exists a neighborhood $U_{p} \in \mathcal{V}(\bar{x})$, such that, for every $x, u \in U_{p}$,

$$
|f(x, p)-f(u, p)| \leq L\|x-u\| .
$$

The proof of the next result is given only for completeness.

Theorem 1.1 Suppose that $f$ is L-Lipschitz at $\bar{x} \in X$ with respect to $M \subset P$ and $\bar{x}$ is solution for $\left(P_{s}\right)$ with respect to $M$. Then for every $p \in M$, there exists a neighborhood $V_{p}$ of $\bar{x}$ s.t. for every $x \in V_{p}$,

$$
f(\bar{x}, p) \leq f(x, p)+L d(x, S(p)) .
$$

Proof. Let $p \in M$. Let $U_{p}$ be a neighborhood of $\bar{x}$ s.t. both relations (1.2) and (1.3) hold. Now the arguments follows as in [3, Proposition 2.4.3]. There exists $\theta>0$ s.t. $B(\bar{x}, \theta) \subset U_{p}$. Consider $V_{p}=B(\bar{x}, \theta / 3)$ and take $x \in V_{p}$. Clearly, if $x \in V_{p}$ and $p \in M$ with $x \in S(p)$, then (1.4) holds from the definition of the solution concept. Consider the situation where $x \in V_{p}, p \in M$, but $(x, p) \notin \operatorname{Gr} H$. Then for every $\varepsilon \in(0, \theta / 3)$ there is $x_{\varepsilon}^{p} \in S(p)$ s.t.

$$
\begin{aligned}
\left\|x-x_{\varepsilon}^{p}\right\| & <d(x, S(p))+\varepsilon \\
& \leq\|x-\bar{x}\|+\varepsilon \\
& \leq \theta / 3+\varepsilon<2 \theta / 3 .
\end{aligned}
$$

Hence,

$$
\begin{aligned}
\left\|x_{\varepsilon}^{p}-\bar{x}\right\| & \leq\left\|x_{\varepsilon}^{p}-x\right\|+\|x-\bar{x}\| \\
& <2 \theta / 3+\theta / 3=\theta
\end{aligned}
$$

Consequently, $x_{\varepsilon}^{p} \in U_{p} \cap S(p)$, so,

$$
\begin{aligned}
f(\bar{x}, p) & \leq f\left(x_{\varepsilon}^{p}, p\right) \leq f(x, p)+L\left\|x-x_{\varepsilon}^{p}\right\| \\
& \leq f(x, p)+L(d(x, S(p))+\varepsilon) \\
& =f(x, p)+L d(x, S(p))+L \varepsilon .
\end{aligned}
$$

Letting $\varepsilon \rightarrow 0$, we obtain the conclusion.

In scalar non-parametric constraint optimization this kind of penalization with a distance function is very useful when one additionally uses a metric regularity property of the constraint system. In our generalized setting the needed regularity is to exist $r>0$ s.t. an inequality of the form

$$
d(x, S(p)) \leq \operatorname{rd}(0, H(x, p))
$$


holds for every $p \in M$ and for all $x$ in a neighborhood of $\bar{x}$. As we shall see in the fourth section, such a relation allows us to work with the initial set-valued map $H$ instead of the implicit set-valued map $S$. The study of this relation is also one the main topics of the present paper and we develop it in the third section. Later on, we shall be back to the optimization problems in order to apply the main results.

Moreover, let us consider another possibility to define a concept of solution for $\left(P_{S}\right)$, corresponding to the case where we think $\left(P_{S}\right)$ as an optimization problem in both variables, the so-called optimization with equilibrium constraints. Namely, one says that $(\bar{x}, \bar{p})$ is a local solution for $\left(P_{S}\right)$ if there exists some neighborhoods $U$ and $W$ of $\bar{x}$ and $\bar{p}$, respectively, s.t. for all $(x, p) \in U \times W$ with $0 \in H(x, p)$, one has:

$$
f(\bar{x}, \bar{p}) \leq f(x, p)
$$

The transformation of this problem with constraints into an unconstrained problem can be done by means of the following result.

Theorem 1.2 Suppose that $f$ is L-Lipschitz at $(\bar{x}, \bar{p}) \in X \times P$ and $(\bar{x}, \bar{p})$ is a local solution for $\left(P_{S}\right)$. Then $(\bar{x}, \bar{p})$ is a local minimum of the scalar function

$$
(x, p) \rightarrow f(x, p)+L d((p, x), \operatorname{Gr} S) .
$$

Proof. Let $U, W$ be the neighborhoods of $\bar{x}$ and $\bar{p}$ s.t. both relations (1.6) and the Lipschitz property hold. There exists $\theta>0$ s.t. $B(\bar{x}, \theta) \times B(\bar{p}, \theta) \subset U \times W$. Consider $(x, p) \in B(\bar{x}, \theta / 6) \times$ $B(\bar{p}, \theta / 6)$. If $0 \in H(x, p)$ (i.e. $x \in S(p))$ then one obviously has

$$
f(\bar{x}, \bar{p})+L d((\bar{p}, \bar{x}), \operatorname{Gr} S)=f(\bar{x}, \bar{p}) \leq f(x, p)+L d((p, x), \operatorname{Gr} S)=f(x, p) .
$$

Consider the situation where $(x, p) \in B(\bar{x}, \theta / 6) \times B(\bar{p}, \theta / 6)$, but $(x, p, 0) \notin \operatorname{Gr} H$. Then for every $\varepsilon \in(0, \theta / 3)$ there is $\left(p_{\varepsilon}, x_{\varepsilon}\right) \in \operatorname{Gr} S$ s.t.

$$
\begin{aligned}
\left\|(x, p)-\left(x_{\varepsilon}, p_{\varepsilon}\right)\right\| & <d((p, x), \operatorname{Gr} S)+\varepsilon \\
& \leq\|(x, p)-(\bar{x}, \bar{p})\|+\varepsilon \\
& \leq \theta / 3+\varepsilon<2 \theta / 3 .
\end{aligned}
$$

Therefore,

$$
\begin{aligned}
\left\|\left(x_{\varepsilon}, p_{\varepsilon}\right)-(\bar{x}, \bar{p})\right\| & \leq\left\|\left(x_{\varepsilon}, p_{\varepsilon}\right)-(x, p)\right\|+\|(x, p)-(\bar{x}, \bar{p})\| \\
& <2 \theta / 3+\theta / 3=\theta .
\end{aligned}
$$

Consequently, $\left(x_{\varepsilon}, p_{\varepsilon}\right) \in(U \times W) \cap \operatorname{Gr} S^{-1}$, so,

$$
\begin{aligned}
f(\bar{x}, \bar{p}) & \leq f\left(x_{\varepsilon}, p_{\varepsilon}\right) \leq f(x, p)+L\left\|(x, p)-\left(x_{\varepsilon}, p_{\varepsilon}\right)\right\| \\
& \leq f(x, p)+L(d((p, x), \operatorname{Gr} S)+\varepsilon) \\
& =f(x, p)+L d((p, x), \operatorname{Gr} S)+L \varepsilon .
\end{aligned}
$$

Letting $\varepsilon \rightarrow 0$, we obtain the conclusion.

In this case, in order to get reasonable optimality conditions, one needs to avoid the implicit multifunction $S$, therefore one imposes graphical regularity: to exist $r>0$ s.t. an inequality of the form

$$
d((p, x), \operatorname{Gr} S) \leq \operatorname{rd}((x, p, 0), \operatorname{Gr} H)
$$

holds for all $(x, p)$ in a neighborhood of $(\bar{x}, \bar{p})$.

This relation is studied, together with (1.5), in Section 3. Note that in [10] the Clarke penalization is used together with some other inequalities in order to get optimality conditions. 


\section{Concepts and tools}

Most of the results of this paper work for several types of generalized differentiation objects as we shall made precise later. But, for the clarity of our discussion we mainly use the constructions developed by Mordukhovich and his collaborators (see [12]). We briefly remind these concepts and results. Firstly, recall that $X^{*}$ denotes the topological dual of the Banach space $X$, while the symbol $w^{*}$ is used for the weak-star topology of the dual system $\left(X, X^{*}\right)$.

Definition 2.1 Let $S$ be a non-empty subset of $X$ and let $x \in S, \varepsilon \geq 0$. The set of $\varepsilon-$ normals to $S$ at $x$ is

$$
\widehat{N}_{\varepsilon}(S, x):=\left\{x^{*} \in X^{*} \mid \underset{u S_{x}}{\limsup } \frac{x^{*}(u-x)}{\|u-x\|} \leq \varepsilon\right\} .
$$

If $\varepsilon=0$, the elements in the right-hand side of (2.1) are called Fréchet normals and their collection, denoted by $\widehat{N}(S, x)$, is the Fréchet normal cone to $S$ at $x$.

Let $\bar{x} \in S$. The basic (or limiting, or Mordukhovich) normal cone to $S$ at $\bar{x}$ is

$$
N(S, \bar{x}):=\left\{x^{*} \in X^{*} \mid \exists \varepsilon_{n} \downarrow 0, x_{n} \stackrel{S}{\rightarrow} \bar{x}, x_{n}^{*} \stackrel{w^{*}}{\rightarrow} x^{*}, x_{n}^{*} \in \widehat{N}_{\varepsilon_{n}}\left(S, x_{n}\right), \forall n \in \mathbb{N}\right\}
$$

If $X$ is an Asplund space (i.e. a Banach space where every convex continuous function is generically Fréchet differentiable), the formula for the basic normal cone takes a simpler form, namely:

$$
N(S, \bar{x})=\left\{x^{*} \in X^{*} \mid \exists x_{n} \stackrel{S}{\rightarrow} \bar{x}, x_{n}^{*} \stackrel{w^{*}}{\rightarrow} x^{*}, x_{n}^{*} \in \widehat{N}\left(S, x_{n}\right), \forall n \in \mathbb{N}\right\} .
$$

Let $f: X \rightarrow \overline{\mathbb{R}}$ be finite at $\bar{x} \in X$; the Fréchet subdifferential of $f$ at $\bar{x}$ is the set

$$
\widehat{\partial} f(\bar{x}):=\left\{x^{*} \in X^{*} \mid\left(x^{*},-1\right) \in \widehat{N}(\operatorname{epi} f,(\bar{x}, f(\bar{x})))\right\}
$$

and the basic (or limiting, or Mordukhovich) subdifferential of $f$ at $\bar{x}$ is

$$
\partial f(\bar{x}):=\left\{x^{*} \in X^{*} \mid\left(x^{*},-1\right) \in N(\operatorname{epi} f,(\bar{x}, f(\bar{x})))\right\},
$$

where epi $f$ denotes the epigraph of $f$. On Asplund spaces one has

$$
\partial f(\bar{x})=\limsup _{x \underset{f}{\rightarrow} \bar{x}} \widehat{\partial} f(x),
$$

and, in particular, $\widehat{\partial} f(\bar{x}) \subset \partial f(\bar{x})$. If $f$ is convex, then both these subdifferential do coincide with the classical Fenchel subdifferential. If $\delta_{\Omega}$ denotes the indicator function associated with a nonempty set $\Omega \subset X$ (i.e. $\delta_{\Omega}(x)=0$ if $x \in \Omega, \delta_{\Omega}(x)=\infty$ if $x \notin \Omega$ ), then for any $\bar{x} \in \Omega, \widehat{\partial} \delta_{\Omega}(\bar{x})=\widehat{N}(\Omega, \bar{x})$ and $\partial \delta_{\Omega}(\bar{x})=N(\Omega, \bar{x})$. Let $\Omega \subset X$ be a nonempty closed set and take $\bar{x} \in \Omega$; then one has

$$
N(\Omega, \bar{x})=\bigcup_{\lambda>0} \lambda \partial d(\cdot, \Omega)(\bar{x})
$$

The basic subdifferential satisfies a robust sum rule (see [12, Theorem 3.36]): if $X$ is Asplund, $f_{1}, f_{2}, \ldots, f_{n-1}: X \rightarrow \mathbb{R}$ are Lipschitz around $\bar{x}$ and $f_{n}: X \rightarrow \overline{\mathbb{R}}$ is lower semicontinuous around this point, then

$$
\partial\left(\sum_{i=1}^{n} f_{i}\right)(\bar{x}) \subset \sum_{i=1}^{n} \partial f_{i}(\bar{x})
$$


We shall also need a calculus rule concerning the partial subgradients, that one can find in [12, Corollary 3.44]. We reproduce here this result in the (less general) form that we actually need in the sequel.

Proposition 2.2 Let $X, Y$ be Asplund spaces and $\varphi: X \times Y \rightarrow \overline{\mathbb{R}}=\mathbb{R} \cup\{+\infty\}$ be a Lipschitz function around $(\bar{x}, \bar{y}) \in X \times Y$. Then:

$$
\partial \varphi(\cdot, \bar{y})(\bar{x}) \subset\left\{x^{*} \in X^{*} \mid \exists y^{*} \in Y^{*} \text { with }\left(x^{*}, y^{*}\right) \in \partial \varphi(\bar{x}, \bar{y})\right\} .
$$

Definition 2.3 Let $F: X \rightrightarrows Y$ be a set-valued map and $(\bar{x}, \bar{y}) \in \operatorname{Gr} F$. Then the Fréchet coderivative at $(\bar{x}, \bar{y})$ is the set-valued map $\widehat{D}^{*} F(\bar{x}, \bar{y}): Y^{*} \rightrightarrows X^{*}$ given by

$$
\widehat{D}^{*} F(\bar{x}, \bar{y})\left(y^{*}\right):=\left\{x^{*} \in X^{*} \mid\left(x^{*},-y^{*}\right) \in \widehat{N}(\operatorname{Gr} F,(\bar{x}, \bar{y}))\right\} .
$$

Similarly, the normal coderivative of $F$ at $(\bar{x}, \bar{y})$ is the set-valued map $D_{N}^{*} F(\bar{x}, \bar{y}): Y^{*} \rightrightarrows X^{*}$ given by

$$
D_{N}^{*} F(\bar{x}, \bar{y})\left(y^{*}\right):=\left\{x^{*} \in X^{*} \mid\left(x^{*},-y^{*}\right) \in N(\operatorname{Gr} F,(\bar{x}, \bar{y}))\right\} .
$$

Note that, in fact, the concept of normal coderivative, independently of the normal cone used in its definition, was introduced in [11].

Besides (2.2) we shall need the following formula obtained by Thibault in [17] (see also [14] for further generalizations and details): if $X, Y$ are Banach spaces, $F: X \rightrightarrows Y$ has closed graph and $(\bar{x}, \bar{y}) \in \operatorname{Gr} F$ then

$$
N(\operatorname{Gr} F,(\bar{x}, \bar{y}))=\bigcup_{\lambda>0} \lambda \partial \rho_{F}(\bar{x}, \bar{y}),
$$

where $\rho_{F}: X \times Y \rightarrow \mathbb{R} \cup\{-\infty\}, \rho_{F}(x, y)=d(y, F(x))$.

In order to conclude this part we remind a subdifferential chain rule (see [12, Corollary 3.43]). Recall ([12, Definition 3.25]) that a function $f: X \rightarrow Y$ is said to be strictly Lipschitz at $\bar{x}$ if it is locally Lipschitzian around this point and there exists a neighborhood $V$ of the origin in $X$ s.t. the sequence $\left(t_{k}^{-1}\left(f\left(x_{k}+t_{k} v\right)-f\left(x_{k}\right)\right)\right)_{k \in \mathbb{N}}$ contains a norm convergent subsequence whenever $v \in V, x_{k} \rightarrow \bar{x}, t_{k} \downarrow 0$. Suppose that $X, Y$ are Asplund spaces. Let $f: X \rightarrow Y$ and $\varphi: Y \rightarrow \mathbb{R}$ s.t. $f$ is strictly Lipschitz at $\bar{x} \in X$ and $\varphi$ is Lipschitz around $f(\bar{x})$; then

$$
\partial(\varphi \circ f)(\bar{x}) \subset \bigcup_{y^{*} \in \partial \varphi(f(\bar{x}))} \partial\left(y^{*} \circ f\right)(\bar{x}) .
$$

\section{Regularity of constraint system}

This section is devoted to survey and then to establish several conditions ensuring the inequalities (1.5) and (1.7) with a special emphasis on the case of epigraphical set-valued maps. We look at two different ways to guarantee the desired relations: firstly, we impose topological conditions on $H$ and, secondly, we look after coderivative conditions.

Next, we recall a definition.

Definition 3.1 Let $L>0, H: X \times P \rightrightarrows Y$ be a multifunction, $((\bar{x}, \bar{p}), \bar{y}) \in \mathrm{Gr} H$ and, for every $p \in P$, denote $H_{p}(\cdot):=H(\cdot, p)$. Then $H$ is said to be open at linear rate $L>0$, or $L$-open, with respect to $x$ uniformly in $p$ around $((\bar{x}, \bar{p}), \bar{y})$ if there exist a positive number $\varepsilon>0$ and some 
neighborhoods $U \in \mathcal{V}(\bar{x}), V \in \mathcal{V}(\bar{p}), W \in \mathcal{V}(\bar{y})$ such that, for every $\rho \in(0, \varepsilon)$, every $p \in V$ and every $(x, y) \in \operatorname{Gr} H_{p} \cap[U \times W]$,

$$
B(y, \rho L) \subset H_{p}(B(x, \rho)),
$$

Now, we remind and comment some existing results in this direction. The first result emphasizes the link between the partial linear openness of the multifunction $H$ and the inequalities (1.5) and (1.7). The first part of this theorem is proved in [7] in full Banach spaces framework (but it works also if $P$ is just a topological space), while the second part comes on the same lines as in $[6$, Theorem $5.2]$.

Theorem 3.2 Let $X, Y$ be Banach spaces, $P$ be a topological space, $H: X \times P \rightrightarrows Y$ be a set-valued map which is inner semicontinuous at $(\bar{x}, \bar{p}, 0) \in \mathrm{Gr} H$. Suppose that $H$ is open with linear rate $c>0$ with respect to $x$ uniformly in $p$ around $(\bar{x}, \bar{p}, 0)$. Then there exist $r_{0}>0$ and $U \in \mathcal{V}(\bar{p})$ such that, for every $(x, p) \in B\left(\bar{x}, r_{0}\right) \times U$,

$$
d(x, S(p)) \leq c^{-1} d(0, H(x, p)) .
$$

If, moreover, $P$ is a metric space, then there exist $\bar{r}, \bar{t}>0$ such that, for every $(x, p) \in B(\bar{x}, \bar{r}) \times$ $B(\bar{p}, \bar{t})$,

$$
d((p, x), \operatorname{Gr} S) \leq\left(1+c^{-1}\right) d((x, p, 0), \text { Gr } H) .
$$

The desired inequalities follow as well from the coderivative conditions, as illustrated in the result below proved in [6].

Theorem 3.3 Let $X, Y$ be Asplund spaces, $P$ be a topological space and $H: X \times P \rightrightarrows Y$ be a set-valued map such that $0 \in H(\bar{x}, \bar{p})$. Suppose that the following assumptions are satisfied:

(i) there exists $U_{1} \in \mathcal{V}(\bar{p})$ such that, for every $p \in U_{1}$, Gr $H_{p}$ is closed;

(ii) $H$ is inner semicontinuous at $(\bar{x}, \bar{p}, 0)$;

(iii) there exist $r, s, c>0$ and $U_{2} \in \mathcal{V}(\bar{p})$ such that, for every $p \in U_{2}$, every $(x, y) \in \operatorname{Gr} H_{p} \cap$ $[B(\bar{x}, r) \times B(\bar{y}, s)]$ and every $y^{*} \in Y^{*}, x^{*} \in \widehat{D}^{*} H_{p}(x, y)\left(y^{*}\right)$,

$$
c\left\|y^{*}\right\| \leq\left\|x^{*}\right\|
$$

Then the following are true:

(a) For every $a \in(0, c)$, there exist $U \in \mathcal{V}(\bar{p})$ and $\tau>0$ such that, for every $(x, p) \in B(\bar{x}, \tau) \times U$,

$$
d(x, S(p)) \leq a^{-1} d(0, H(x, p)) .
$$

(b) If, moreover, $P$ is a metric space, there exist $\gamma_{0}>0, \tau_{0}>0$ such that, for every $(x, p) \in$ $B\left(\bar{x}, \tau_{0}\right) \times B\left(\bar{p}, \gamma_{0}\right)$,

$$
d((p, x), \operatorname{Gr} S) \leq\left(1+a^{-1}\right) d((x, p, 0), \text { Gr } H) .
$$

We look now to the special case of epigraphical multifunctions. Remind that, for a multifunction $G: X \rightrightarrows Y$ and a closed convex proper cone $C \subset Y$, the epigraphical multifunction associated with $G$ is $\tilde{G}: X \rightrightarrows Y$ given by $\tilde{G}(x):=G(x)+C$ for every $x \in X$. We denote the dual cone of $C$ by $C^{*}:=\left\{y^{*} \mid y^{*}(y) \geq 0, \forall y \in C\right\}$.

(... de ce se ia ..) 
The next theorem will be used in the sequel and it presents sufficient conditions for the linear openness of the epigraphical multifunction in terms of the Fréchet coderivative of the initial multifunction. Note that the first part is [5, Theorem 3.6], while the second part could be easily obtained by inspecting the proof of the first conclusion.

Theorem 3.4 Let $X, Y$ be Asplund spaces, $G: X \rightrightarrows Y$ be a set-valued map, $C$ be a proper closed convex cone in $Y$ and $(\bar{x}, \bar{y}) \in \operatorname{Gr} G$. Suppose that the following assumptions are satisfied:

(i) $\operatorname{Gr} G$ is locally closed at $(\bar{x}, \bar{y})$;

(ii) there exist $r, c>0$ s.t. for every $(x, y) \in \operatorname{Gr} G \cap[B(\bar{x}, r) \times B(\bar{y}, r)]$ and every $y^{*} \in$ $C^{*} \cap \mathbb{S}_{Y^{*}}, z^{*} \in 2 c \mathbb{B}_{Y^{*}}, x^{*} \in \hat{D}^{*} G(x, y)\left(y^{*}+z^{*}\right)$,

$$
c\left\|y^{*}+z^{*}\right\| \leq\left\|x^{*}\right\|
$$

Then for every $a \in(0, c)$, there exists $\varepsilon>0$ such that, for every $\rho \in(0, \varepsilon]$,

$$
B(\bar{y}, \rho a) \subset G(B(\bar{x}, \rho))+C \cap B(0,(a+1) \rho) \subset \tilde{G}(B(\bar{x}, \rho)) .
$$

If $\operatorname{Gr} G$ is closed, the conclusion is more precise in the following sense: for every $a \in(0, c)$, there exists $\varepsilon:=\min \left(\frac{1}{2}\left(\frac{c}{c+1}-\frac{a}{a+1}\right), \frac{r}{(a+1)}\right)>0$ such that, for every $\rho \in(0, \varepsilon]$,

$$
B(\bar{y}, \rho a) \subset \tilde{G}(B(\bar{x}, \rho)) .
$$

Based on this result, let us now consider in the light of our aim, the case when $H$ is given as an epigraphical multifunction, i.e. $H(x, p)=F(x, p)+C$, for every $(x, p) \in X \times P$, where $C$ is, as above, a proper closed convex cone in $Y$. In a similar way to the technique of proving Theorem 3.3, one can obtain the next theorem, which shows the desired inequalities, which involve now $S$ (the solution map associated to $H$ ) and $F$.

Theorem 3.5 Let $X, Y$ be Asplund spaces, $P$ be a topological space and $F: X \times P \rightrightarrows Y$ be a set-valued map such that $0 \in F(\bar{x}, \bar{p})$. Suppose that the following assumptions are satisfied:

(i) there exists $U_{1} \in \mathcal{V}(\bar{p})$ such that, for every $p \in U_{1}, G r F_{p}$ is closed;

(ii) $F$ is inner semicontinuous at $(\bar{x}, \bar{p}, 0)$;

(iii) there exist $r, c>0$ and $U_{2} \in \mathcal{V}(\bar{p})$ such that, for every $p \in U_{2}$, every $(x, y) \in \operatorname{Gr} F_{p} \cap$ $[B(\bar{x}, r) \times B(0, r)]$ and every $y^{*} \in \mathbb{S}_{Y^{*}} \cap C^{*}$, every $z^{*} \in 2 c \mathbb{B}_{Y^{*}}$, and every $x^{*} \in \widehat{D}^{*} F_{p}(x, y)\left(y^{*}+z^{*}\right)$,

$$
c\left\|y^{*}+z^{*}\right\| \leq\left\|x^{*}\right\|
$$

Then for every $a \in(0, c)$, there exist $U \in \mathcal{V}(\bar{p})$ and $\tau>0$ such that, for every $(x, p) \in B(\bar{x}, \tau) \times U$,

$$
d(x, S(p)) \leq a^{-1} d(0, F(x, p)) .
$$

If, moreover, $P$ is a metric space, then for every $a \in(0, c)$, there exist $\tau_{0}>0, \gamma_{0}>0$ such that, for every $(x, p) \in B\left(\bar{x}, \tau_{0}\right) \times B\left(\bar{p}, \gamma_{0}\right)$,

$$
d((p, x), \operatorname{Gr} S) \leq\left(1+a^{-1}\right) d((x, p, 0), \operatorname{Gr} F) .
$$


Proof. Concerning the proof of (3.6), fix arbitrary $a \in(0, c)$ and $\rho \in\left(0, \min \left(\frac{1}{2}\left(\frac{c}{c+1}-\frac{a}{a+1}\right), \frac{r}{2(a+1)}\right)\right)$. Using the inner semicontinuity of $F$ at $(\bar{x}, \bar{p})$, we can find $U_{0} \in \mathcal{V}(\bar{p})$ and $\nu>0$ such that for every $(x, p) \in B(\bar{x}, \nu) \times U_{0}$,

$$
F(x, p) \cap B(0, a \rho) \neq \emptyset .
$$

Denote $U:=U_{0} \cap U_{1} \cap U_{2}, \tau:=\min \left(\nu, \frac{r}{2}\right)$ and take $(x, p) \in B(\bar{x}, \tau) \times U$.

If $0 \in F(x, p) \subset H(x, p)$, then (3.6) trivially holds. Suppose that $0 \notin F(x, p)$ and then, for every $\varepsilon>0$, we can find $y_{\varepsilon} \in F(x, p)$ such that

$$
\left\|y_{\varepsilon}\right\|<d(0, F(x, p))+\varepsilon
$$

Because from (3.8) we have that $d(0, F(x, p))<a \rho$, we can take $\varepsilon>0$ sufficiently small such that $d(0, F(x, p))+\varepsilon<a \rho$. Using $(3.9)$, we have that

$$
0 \in B\left(y_{\varepsilon}, d(0, F(x, p))+\varepsilon\right) \subset B\left(y_{\varepsilon}, a \rho\right) .
$$

Moreover,

$$
\begin{aligned}
B\left(x, 2^{-1} r\right) & \subset B(\bar{x}, r), \\
B\left(y_{\varepsilon}, 2^{-1} r\right) & \subset B\left(0,2^{-1} r+a \rho\right) \subset B(0, r) .
\end{aligned}
$$

Hence we can apply Theorem 3.4 for $\left(x, y_{\varepsilon}\right) \in \operatorname{Gr} F_{p}, r_{0}:=2^{-1} r$, and $\rho_{0}:=\frac{1}{a}(d(0, F(x, p))+\varepsilon)<$ $\rho$, showing that

$$
B\left(y_{\varepsilon}, a \rho_{0}\right) \subset F_{p}\left(B\left(x, \rho_{0}\right)\right)+C=H_{p}\left(B\left(x, \rho_{0}\right)\right) .
$$

We can find then $\widetilde{x} \in B\left(x, \rho_{0}\right)$ such that $y \in H_{p}(\widetilde{x})$, or $\widetilde{x} \in S(p)$. Hence

$$
d(x, S(p)) \leq\|x-\widetilde{x}\|<\frac{1}{a}(d(y, F(x, p))+\varepsilon) .
$$

Making $\varepsilon \rightarrow 0$, we obtain (3.6).

Let us now prove (3.7). Take as above $a \in(0, c)$ and $\rho \in\left(0, \min \left(\frac{1}{2}\left(\frac{c}{c+1}-\frac{a}{a+1}\right), \frac{r}{4(a+1)}\right)\right)$, use again the inner semicontinuity of $F$ at $(\bar{x}, \bar{p})$ and find the neighborhood $U_{0}$ of $\bar{p}$ and $\nu>0$ such that for every $(x, p) \in B(\bar{x}, \nu) \times U_{0}$, (3.8) holds. If $P$ is a metric space, we can find $\gamma>0$ such that $B(\bar{p}, \gamma) \subset U_{0} \cap U_{1} \cap U_{2}$. Take $\tau_{0}:=\min \left(\frac{\gamma}{3}, \nu, \frac{r}{4}\right), \gamma_{0}:=\frac{\gamma}{3}$ and choose $(x, p) \in B\left(\bar{x}, \tau_{0}\right) \times B\left(\bar{p}, \gamma_{0}\right)$.

We have that

$$
\begin{aligned}
& d((x, p, 0), \operatorname{Gr} F) \leq\|x-\bar{x}\|+d(p, \bar{p})<\frac{\gamma}{3}+\frac{\gamma}{3}=\frac{2 \gamma}{3}, \\
& d((x, p, 0), \operatorname{Gr} F)<d(0, F(x, p))<a \rho .
\end{aligned}
$$

Without loss of generality suppose that $0 \notin F(x, p)$, hence for every $\varepsilon>0$ sufficiently small such that $d((x, p, 0), \operatorname{Gr} F)+\varepsilon<\min \left(a \rho, \frac{2 \gamma}{3}\right)$ we can find $\left(x_{\varepsilon}, p_{\varepsilon}, y_{\varepsilon}\right) \in \operatorname{Gr} F$ satisfying

$$
\begin{aligned}
\max \left(\left\|y_{\varepsilon}\right\|, d\left(p_{\varepsilon}, p\right)\right) & \leq\left\|y_{\varepsilon}\right\|+\left\|x_{\varepsilon}-x\right\|+d\left(p_{\varepsilon}, p\right) \\
& <d((x, p, 0), \operatorname{Gr} F)+\varepsilon .
\end{aligned}
$$

Hence,

$$
\begin{aligned}
p_{\varepsilon} & \in B\left(p, \frac{2 \gamma}{3}\right) \subset B(\bar{p}, \gamma), \\
0 & \in B\left(y_{\varepsilon}, d((x, p, 0), \text { Gr } F)+\varepsilon\right) \subset B\left(y_{\varepsilon}, a \rho\right)
\end{aligned}
$$


and

$$
\begin{aligned}
& B\left(x_{\varepsilon}, 4^{-1} r\right) \subset B\left(x, 4^{-1} r+a \rho\right) \subset B\left(x, 2^{-1} r\right) \subset B(\bar{x}, r), \\
& B\left(y_{\varepsilon}, 2^{-1} r\right) \subset B\left(0,2^{-1} r+a \rho\right) \subset B(0, r) .
\end{aligned}
$$

Then we can apply Theorem 3.4 for $\left(x_{\varepsilon}, p_{\varepsilon}, y_{\varepsilon}\right)$ such that $y_{\varepsilon} \in F_{p_{\varepsilon}}\left(x_{\varepsilon}\right), r^{\prime}:=4^{-1} r$ and $\rho^{\prime}:=$ $\frac{1}{a}(d((x, p, 0), \operatorname{Gr} F)+\varepsilon)<\rho$ and, using (3.11), we obtain that

$$
0 \in B\left(y_{\varepsilon}, a \rho^{\prime}\right) \subset F_{p_{\varepsilon}}\left(B\left(x_{\varepsilon}, \rho^{\prime}\right)\right)+C \cap B(0,(a+1) \rho) \subset H_{p_{\varepsilon}}\left(B\left(x_{\varepsilon}, \rho^{\prime}\right)\right) .
$$

Then we have that there exists $\widetilde{x} \in B\left(x_{\varepsilon}, \rho^{\prime}\right)$ such that $0 \in H_{p_{\varepsilon}}(\widetilde{x})$, or $\left(\widetilde{x}, p_{\varepsilon}\right) \in \operatorname{Gr} S$. Hence, using also (3.10),

$$
\begin{aligned}
d((p, x), \operatorname{Gr} S) & \leq\|\widetilde{x}-x\|+d\left(p_{\varepsilon}, p\right) \\
& \leq\left\|\widetilde{x}-x_{\varepsilon}\right\|+\left\|x_{\varepsilon}-x\right\|+d\left(p_{\varepsilon}, p\right) \\
& <\frac{1}{a}(d((x, p, 0), \operatorname{Gr} F)+\varepsilon)+d((x, p, 0), \operatorname{Gr} F)+\varepsilon
\end{aligned}
$$

Making again $\varepsilon \rightarrow 0$, we obtain (3.7).

\section{Applications}

We come back to our motivational facts exposed in the first section. We have previously seen, on one hand, how regularity of parametric systems could be used for transformation of a constraint problem into an unconstrained one by means of penalization and, on the other hand, how the regularity could be obtained in different ways by different types of conditions. In this section we are going to apply the penalization in order to get optimality conditions for several solution concepts in parametric solid vector optimization. Before starting, we refer to the recent works [13] and [1] where similar problems were considered in some greater generality. Our aim here is to sample how to get necessary optimality conditions under metrical and graphical regularity and, in order to keep the accent on metrical conditions, we restrict the attention to the solid case (i.e. the case where the ordering cone has nonempty topological interior).

Let $g: X \times P \rightarrow Z$ be a parametric vector valued function taking values into the Banach space $Z$ ordered by a closed convex pointed cone $K$ with nonempty interior (i.e. int $K \neq \emptyset$ ). As usual, the order $\leq_{K}$ on $Z$ associated to the cone $K$ is given by the equivalence $x \leq_{K} y$ if and only if $y-x \in K$. We recall that if $R \subset Z$ is a nonempty set, then a point $r \in R$ is called weak minimal point for $R$ with respect to $K$ if

$$
(R-r) \cap-\operatorname{int} K=\emptyset .
$$

Keeping the other notations from previous sections, the problem we propose is as follows

$$
\left(P_{V}\right): \min g(x, p) \text { subject to } 0 \in H(x, p) \text {, }
$$

where "min" has a double meaning based on the notion of weak minimal point, as follows.

Let $M \subset P$ be a nonempty set. We say that $\bar{x} \in X$ is a weak solution for $\left(P_{V}\right)$ with respect to $M$ if $\bar{x} \in \bigcap_{p \in M} S(p)$ and for every $p \in M$ there is an $\varepsilon_{p}>0$ s.t. for every $x \in B(\bar{x}, \varepsilon) \cap S(p)$, one has

$$
g(x, p)-g(\bar{x}, p) \notin-\operatorname{int} K .
$$


The other definition of solution runs as follows: we say that $(\bar{x}, \bar{p})$ is a local weak solution for $\left(P_{V}\right)$ if there exists some neighborhoods $U$ and $W$ of $\bar{x}$ and $\bar{p}$, respectively, s.t. for all $(x, p) \in U \times W$ with $0 \in H(x, p)$, one has:

$$
g(x, p)-g(\bar{x}, \bar{p}) \notin-\operatorname{int} K .
$$

Once again, we say that $g$ is $L$-Lipschitz at $\bar{x}$ with respect to $M \subset P$ if relation (1.3) holds for $g$ with the norm instead of modulus in the left-hand side. The main tool we use in conjunction with the coderivative calculus is contained in the next results which is proved, even in a more general setting, in [8]. In this result $\partial$ denotes the Fenchel subdifferential of a convex function and $\mathrm{bd}(K)$ denotes the topological boundary of $K$.

Theorem 4.1 Let $K \subset Z$ be a closed convex cone with nonempty interior. Then for every $e \in \operatorname{int} K$ the functional $s_{e}: Z \rightarrow \mathbb{R}$ given by

$$
s_{e}(z)=\inf \{\lambda \in \mathbb{R} \mid \lambda e \in z+K\}
$$

is continuous, sublinear, strictly-int $K$-monotone and:

(i) $\partial s_{e}(0)=\left\{v^{*} \in K^{*} \mid v^{*}(e)=1\right\}$;

(ii) for every $u \in Z, \partial s_{e}(u) \neq \emptyset$ and

$$
\partial s_{e}(u)=\left\{v^{*} \in K^{*} \mid v^{*}(e)=1, v^{*}(u)=s_{e}(u)\right\} .
$$

Moreover, $s_{e}$ is $d(e, \operatorname{bd}(K))^{-1}-$ Lipschitz and for every $u \in Z$ and $v^{*} \in \partial s_{e}(u),\|e\|^{-1} \leq\left\|v^{*}\right\| \leq$ $d(e, \operatorname{bd}(K))^{-1}$.

If $A \subset Z$ is a nonempty set s.t. $A \cap(-\operatorname{int} K)=\emptyset$, then $s_{e}(a) \geq 0$ for every $a \in A$.

For $e \in \operatorname{int} K$ we shall denote $d(e, \operatorname{bd}(K))^{-1}$ by $L_{e}$ (the Lipschitz constant for $s_{e}$ ).

We present now the first result of this section.

Theorem 4.2 Suppose that $X, Y, Z, P$ are Asplund spaces. Suppose that $g$ is L-strictly Lipschitz at $\bar{x} \in X$ with respect to $M \subset P$ and $\bar{x}$ is a weak solution for $\left(P_{V}\right)$ with respect to $M$. Moreover, suppose that, for every $p \in M,(1.5)$ holds, $H_{p}$ has closed graph and $H$ is Lipschitz-like around $(\bar{x}, p, 0)$. Then for every $e \in \operatorname{int} K$ and for every $p \in M$ there exist $z^{*} \in K^{*}, z^{*}(e)=1$ and $y^{*} \in Y^{*}$ s.t.

$$
0 \in \partial\left(z^{*} \circ g(\cdot, p)\right)(\bar{x})+D^{*} H_{p}(\bar{x}, 0)\left(y^{*}\right) .
$$

Proof. Take $e \in \operatorname{int} K$ and $p \in M$. Firstly, observe that the scalar application $(x, p) \mapsto s_{e}(g(x, p)-$ $g(\bar{x}, p))$ is $L \cdot L_{e}$-Lipschitz at $\bar{x} \in X$ with respect to $M \subset P$ because for any $u$ in an appropriate neighborhood of $\bar{x}$ one has

$$
\begin{aligned}
\left|s_{e}(g(x, p)-g(\bar{x}, p))-s_{e}(g(u, p)-g(\bar{x}, p))\right| & \leq L_{e}\|g(x, p)-g(u, p)\| \\
& \leq L L_{e}\|x-u\| .
\end{aligned}
$$

Moreover, since $\bar{x}$ is a weak solution for $\left(P_{V}\right)$ with respect to $M$ and taking into account the last conclusion of Theorem 4.1 one deduces that $\bar{x}$ is a solution for

$$
\min s_{e}(g(x, p)-g(\bar{x}, p)) \text { subject to } 0 \in H(x, p) \text {, }
$$


with respect to $M$. Note that, for any $p \in M$, the value of this problem is 0 . Whence, following Theorem 1.1, there exists a neighborhood $V_{p}$ of $\bar{x}$ s.t. for every $x \in V_{p}$,

$$
0 \leq s_{e}(g(x, p)-g(\bar{x}, p))+L L_{e} d(x, S(p))
$$

Now, eventually taking a smaller neighborhood of $\bar{x}$ (denoted $V_{p}$ as well) and using (1.5) one gets that for every $x \in V_{p}$,

$$
0 \leq s_{e}(g(x, p)-g(\bar{x}, p))+L L_{e} r d(0, H(x, p)) .
$$

Taking into account that for $x=\bar{x}$ the right-hand side of the above problem is 0 , one deduces that $\bar{x}$ is a local minimum of the scalar function

$$
x \mapsto s_{e}(g(x, p)-g(\bar{x}, p))+L L_{e} r d(0, H(x, p)) .
$$

Consequently, by the generalized differentiation calculus rules,

$$
0 \in \partial\left(s_{e}(g(\cdot, p)-g(\bar{x}, p))+L L_{e} r d(0, H(\cdot, p))\right)(\bar{x}),
$$

and because the first function is Lipschitz while the second one is lower semicontinuous around the reference point $\bar{x}$ (taking into account that $H$ is Lipschitz-like around $(\bar{x}, p, 0)$ ), we can employ the calculus rule (2.3) in order to write

$$
0 \in \partial s_{e}(g(\cdot, p)-g(\bar{x}, p))(\bar{x})+L L_{e} r \partial d(0, H(\cdot, p))(\bar{x}) .
$$

Since $s_{e}$ is Lipschitz and $g(\cdot, p)$ is strictly Lipschitz, one can apply $(2.5)$ to get

$$
0 \in \bigcup_{z^{*} \in \partial s_{e}(0)} \partial\left(z^{*} \circ g(\cdot, p)\right)(\bar{x})+L L_{e} r \partial d(0, H(\cdot, p))(\bar{x}),
$$

i.e. there exists $z^{*} \in K^{*}, z^{*}(e)=1$ s.t.

$$
0 \in \partial\left(z^{*} \circ g(\cdot, p)\right)(\bar{x})+L L_{e} r \partial d(0, H(\cdot, p))(\bar{x}),
$$

Note that, from the Lipschitz-like property of $H_{p}$, the scalar function $d(\cdot, H(\cdot, p))$ is Lipschitz at $(\bar{x}, 0)$ (see [16, Section 2]) and one uses Proposition 2.2 and relation (2.2) to get

$$
\left.L L_{e} r \partial d(0, H(\cdot, p))(\bar{x}) \subset L L_{e} r\left\{x^{*} \in X^{*} \mid \exists u^{*} \in Y^{*} \text { with }\left(x^{*}, u^{*}\right) \in \partial \rho_{H_{p}}(\bar{x}, 0)\right\} \cdot\right\}
$$

Taking into account (2.4) one concludes the proof.

We pass now to the other kind of vector solution we have introduced before.

Theorem 4.3 Suppose that $X, Y, Z, P$ are Asplund spaces. Suppose that $g$ is L-strictly Lipschitz at $(\bar{x}, \bar{p})$ which is a local weak solution for $\left(P_{V}\right)$. Moreover, suppose that (1.7) holds and $H$ has closed graph. Then for every $e \in \operatorname{int} K$ there exist $z^{*} \in K^{*}, z^{*}(e)=1, y^{*} \in Y^{*}$ s.t.

$$
(0,0) \in \partial\left(z^{*} \circ g(\cdot, \cdot)\right)(\bar{x}, \bar{p})+D^{*} H(\bar{x}, \bar{p}, 0)\left(y^{*}\right) .
$$


Proof. The proof runs along some similar lines as above. We point out the main parts. Take $e \in$ int $K$. The scalar application $(x, p) \mapsto s_{e}(g(x, p)-g(\bar{x}, \bar{p}))$ is $L \cdot L_{e}$-Lipschitz at $(\bar{x}, \bar{p})$. Moreover, one has that $(\bar{x}, \bar{p})$ is a solution for

$$
\min s_{e}(g(x, p)-g(\bar{x}, \bar{p})) \text { subject to } 0 \in H(x, p) .
$$

Following Theorem 1.2, $(\bar{x}, \bar{p})$ is a local solution for the unconstrained problem

$$
\min \left[s_{e}(g(x, p)-g(\bar{x}, \bar{p}))+L L_{e} d((p, x), \operatorname{Gr} S)\right] .
$$

Using (1.7) one gets that $(\bar{x}, \bar{p})$ is a local solution for (still unconstrained) problem

$$
\min \left[s_{e}(g(x, p)-g(\bar{x}, \bar{p}))+L L_{e} r d((x, p, 0), \text { Gr } H)\right] .
$$

The subdifferential rules applied for the sum of two Lipschitz functions gives

$$
(0,0) \in \partial s_{e}(g(\cdot, \cdot)-g(\bar{x}, \bar{p}))(\bar{x}, \bar{p})+L L_{e} r \partial d((\cdot, \cdot, 0), \operatorname{Gr} H)(\bar{x}, \bar{p}) .
$$

Since $s_{e}$ is Lipschitz and $g(\cdot, p)$ is strictly Lipschitz one can apply (2.5). Taking into account (2.4) one gets

$$
(0,0) \in \bigcup_{z^{*} \in \partial s_{e}(0)} \partial\left(z^{*} \circ g\right)(\bar{x}, \bar{p})+L L_{e} r \partial d((\cdot, \cdot, 0), \text { Gr } H)(\bar{x}, \bar{p}),
$$

i.e. there exists $z^{*} \in K^{*}, z^{*}(e)=1$ s.t.

$$
(0,0) \in \partial\left(z^{*} \circ g\right)(\bar{x}, \bar{p})+L L_{e} r \partial d((\cdot, \cdot, 0), \operatorname{Gr} H)(\bar{x}, \bar{p}) \neq \emptyset .
$$

Now, taking into account that the distance function is 1-Lipschitz, following Proposition 2.2,

$$
\partial d((\cdot, \cdot, 0), \operatorname{Gr} H)(\bar{x}, \bar{p}) \subset\left\{\left(x^{*}, p^{*}\right) \mid \exists u^{*} \in Y^{*} \text { with }\left(x^{*}, p^{*}, u^{*}\right) \in \partial d_{\mathrm{Gr} H}(\bar{x}, \bar{p}, 0)\right\} .
$$

But, from (2.2),

$$
\mathbb{R}_{+} \partial d_{\mathrm{Gr} H}(\bar{x}, \bar{p}, 0) \subset N(\operatorname{Gr} H,(\bar{x}, \bar{p}, 0))
$$

whence, replacing $u^{*}$ by $-y^{*}$

$$
(0,0) \in \partial\left(z^{*} \circ g\right)(\bar{x}, \bar{p})+D^{*} H(\bar{x}, \bar{p}, 0)\left(y^{*}\right),
$$

and this concludes the proof.

Note that in the case where $g$ is taken as a scalar function (see the framework and notations of Section 1), then one does not need to scalarize the function and, consequently, the Lipschitz property is enough. We would like also to mention that in this situation one does not need all the calculus rules we quote for Mordukhovich differentiation and, consequently, this result could be formulated as well for many other subdifferential and related coderivatives: see [8] for further details and comments. However, for completeness, we present such a result.

Theorem 4.4 Suppose that $X, Y, Z, P$ are Asplund spaces. Suppose that $f$ is L-Lipschitz at $(\bar{x}, \bar{p})$, which is a local weak solution for $\left(P_{S}\right)$. Moreover, suppose that (1.7) holds and that $H$ has closed graph. Then there exists $y^{*} \in Y^{*}$ s.t.

$$
(0,0) \in \partial f(\bar{x}, \bar{p})+D^{*} H(\bar{x}, \bar{p}, 0)\left(y^{*}\right) .
$$

We conclude by saying that, in view of the schema displayed in this paper, every result on regularity of the constraint system would bring necessary optimality conditions for various types of nonsmooth scalar and vector programs. 


\section{References}

[1] T. Q. Bao, P. Gupta, B. S. Mordukhovich, Necessary conditions for multiobjective optimization with equilibrium constraints, Journal Optimization Theory and Applications 135 (2007), 179203.

[2] T. D. Chuong, J. C. Yao, Coderivatives of efficient point multifunctions in parametric vector optimization, Taiwanese Journal of Mathematics, 13 (2009), 1671-1693.

[3] F. H. Clarke, Optimization and Nonsmooth Analysis, Wiley, New York, 1983.

[4] A. L. Dontchev, R. T. Rockafellar, Implicit functions and solution mappings, Springer, Berlin, 2009 .

[5] M. Durea, R. Strugariu, On some Fermat rules for set-valued optimization problems, Optimization, DOI: 10.1080/02331930903531527.

[6] M. Durea, R. Strugariu, Quantitative results on openness of set-valued mappings and implicit multifunction theorems, Pacific Journal of Optimization, 6 (2010), 533-549.

[7] M. Durea, R. Strugariu, Openness stability and implicit multifunction theorems. Applications to variational systems, submitted.

[8] M. Durea, C. Tammer, Fuzzy necessary optimality conditions for vector optimization problems, Optimization, 58 (2009), 449-467.

[9] A. Göpfert, H. Riahi, C. Tammer, C. Zălinescu, Variational Methods in Partially Ordered Spaces, Springer, Berlin, 2003.

[10] G. Liu, J. Ye, J. Zhu, Partial Exact Penalty for Mathematical Programs with Equilibrium Constraints, Set-Valued Analysis, 16 (2008), 785-804.

[11] B. S. Mordukhovich, Metric approximations and necessary optimality conditions for general classes of extremal problems, Soviet. Math. Dokl., 22 (1980), 526-530.

[12] B. S. Mordukhovich, Variational Analysis and Generalized Differentiation, Vol. I: Basic Theory, Vol. II: Applications, Springer, Grundlehren der mathematischen Wissenschaften (A Series of Comprehensive Studies in Mathematics), Vol. 330 and 331, Berlin, 2006.

[13] B. S. Mordukhovich, Multiobjective optimization problems with equilibrium constraints, Mathematical Programming 117 (2009), 331-354.

[14] B. S. Mordukhovich, N. M. Nam, Subgradient of distance functions with applications to Lipschitzian stability, Mathematical Programming, Ser. B, 104 (2005), 635-668.

[15] S.M. Robinson, Strongly regular generalized equations, Mathematics of Operations Research, 5 (1980), 43-62.

[16] R. T. Rockafellar, Lipschitzian properties of multifunctions, Nonlinear Analysis: Theory Methods and Applications, 9 (1985), 867-885.

[17] L. Thibault, On subdifferentials of optimal value functions, SIAM Journal on Control and Optimization, 29 (1991), 1019-1036. 